\title{
Adquisición y la transferencia de conocimiento tácito de mercadotecnia en pequeños y medianos hoteles
}

\author{
Acquisition and transfer of tacit knowledge of marketing in small \\ and medium hotels
}

\author{
José Luis Zapata Sánchez ${ }^{1, *}$, Judith Cavazos Arroyo² \\ y Yésica Mayett Moreno ${ }^{2}$ \\ ${ }^{1}$ Universidad de Quintana Roo, México \\ ${ }^{2}$ Universidad Popular Autónoma del Estado de Puebla, México
}

Recibido el 15 de julio de 2016; aceptado el 23 de mayo de 2017

Disponible en Internet el 19 de marzo de 2019

\begin{abstract}
Resumen
Esta investigación tuvo como objetivo analizar el efecto de la capacidad de absorción, una cultura abierta de colaboración, confianza entre compañeros de trabajo y la riqueza de medios de comunicación sobre la adquisición de conocimientos tácitos de mercadotecnia, así como el impacto de ésta sobre la transferencia de conocimientos tácitos de mercadotecnia. Entre los empleados de hoteles pequeños y medianos de Playa del Carmen, Quintana Roo, se realizó una investigación cuantitativa y transversal, aplicándose una encuesta personal a 229 empleados seleccionados por conveniencia, que trabajan en el sector hotelero. Para analizar el efecto de las variables en la transferencia de conocimiento tácito de mercadotecnia se utilizó la técnica estadística de ecuaciones estructurales. Los resultados mostraron que la confianza en compañeros de trabajo y la riqueza de medios de comunicación impactan directa y positivamente la adquisición de conocimiento tácito de mercadotecnia y a su vez, ésta a la transferencia de conocimiento tácito de mercadotecnia. Las implicaciones teóricas y aplicadas de los resultados fueron discutidos.
\end{abstract}

Código JEL: D83, M31, J24, C3.

Palabras clave: Conocimiento tácito, Transferencia de conocimiento, Mercadotecnia, Talento humano.

\footnotetext{
*Autor para correspondencia.

Correo electrónico: jluiszap@gmail.com (J. L. Zapata Sánchez)

La revisión por pares es responsabilidad de la Universidad Nacional Autónoma de México
} 


\begin{abstract}
The aim of this research was to analyze the effect of absorption capacity, an open culture of collaboration, trust between co-workers and communication media richness on the acquisition of tacit marketing knowledge, as well as its impact on tacit marketing knowledge transfer. A quantitative and cross-sectional research was carried out among the employees of small and medium-sized hotels in Playa del Carmen, Quintana Roo, applying a personal survey to 229 employees selected for convenience, who work in the hotel sector. The statistical technique of structural equations was used to analyze the effect of variables on the transfer of tacit marketing knowledge. The results showed that trust in co-workers and communication media richness have a direct and positive impact on the acquisition of tacit marketing knowledge and, in turn, on the transfer of tacit marketing knowledge. The theoretical and applied implications of the results were discussed.
\end{abstract}

JEL Classification: D83, M31, J24, C3.

Keywords: Tacit knowledge; Knowledge transfer; Marketing; Human talent.

\title{
Introducción
}

La transferencia de conocimiento es clave para la creación social de conocimiento (Collins y Smith, 2006; Nahapiet, y Ghoshal, 1998). En este sentido, algunas investigaciones recientes sostienen que el desarrollo de un potencial innovador requiere que la empresa sea capaz de combinar adecuadamente las prácticas de gestión de recursos humanos para desarrollar una orientación hacia el aprendizaje que legitime el valor del conocimiento y su intercambio en la organización (Ma, Choi, y O’Connor, 2016). La creación de conocimiento ocurre, por tanto, a través de la transformación en nuevo conocimiento de la percepción e ideas existentes, y las prácticas de recursos humanos favorecen habilidades, motivación y oportunidades del empleado para acceder y movilizar su conocimiento hacia otros empleados, a través de su vínculo, transformación y ampliación en nuevo conocimiento e ideas (Kang, Morris, y Snell, 2007). Por tanto, la transferencia de conocimiento ejerce una armonía parcial en la relación entre las prácticas de gestión de recursos humanos y la incubación de conocimiento.

Aunque la transferencia del conocimiento sea un componente importante de la gestión del conocimiento (Davenport y Prusak, 2000), ésta ha recibido una atención mínima por parte de investigadores y de la comunidad empresarial en lo que respecta a la transferencia de conocimiento tácito de mercadotecnia; involucra un proceso por el cual los empleados con conocimientos en la disciplina transfieren a otros lo que han aprendido, conformando con ello una posible fuente de ventaja competitiva sostenible para la compañía (Ambrosini y Bowman, 2001). Por ello, se ha recomendado profundizar en las variables que impactan en la transferencia de conocimiento de mercadotecnia (Mohamad, Ramayah, y Hathaivaseawong, 2010), especialmente en la industria de la hospitalidad, donde predomina el conocimiento tácito (Cooper, 2006).

México se ha ubicado entre los principales destinos turísticos por llegada (Sistema Integral de Información de Mercados Turísticos [SIIMT], 2014) y la industria del turismo ha generado millones de empleos directos e indirectos en el país (Instituto Nacional de Estadística e Informática [INEGI], 2013; Secretaría de Turismo [SECTUR], 2014). Sin embargo, las micro, pequeñas y medianas empresas (Mipymes) hoteleras, necesitan información especializada para ser competitivas. Así, buscando aportar conocimientos teóricos y aplicados relacionados con este 
sector, esta investigación tiene como objetivo determinar el efecto de la capacidad de absorción, una cultura abierta de colaboración, la confianza entre compañeros de trabajo y la riqueza de medios de comunicación en la adquisición de conocimientos tácitos intraorganizacionales de mercadotecnia y ésta sobre la transferencia de conocimientos tácitos de mercadotecnia, entre los empleados de hoteles pequeños y medianos localizados en la ciudad de Playa del Carmen, Quintana Roo, en México.

\section{Revisión de literatura y planteamiento de hipótesis}

\section{El enfoque del conocimiento tácito de mercadotecnia}

El conocimiento puede ser dividido en dos tipos de acuerdo a su capacidad para ser estructurado y codificado, de tal manera que el conocimiento explícito es considerado científico y altamente estructurado, en cambio, el conocimiento tácito es subjetivo, basado en la experiencia, la intuición y el juicio (Nonaka y Takeuchi, 1995; Gummesson, 2017a). La interacción de ambos tipos de conocimiento conforma el conocimiento organizacional (Easterby-Smith y Prieto, 2008) y la sabiduría pragmática (Gummesson, 2017b).

Diversos estudios han encontrado que una gran cantidad de conocimientos de marketing son tácitos, encarnados en las personas, y por lo tanto difíciles de transferir (Bennett, 1999; Simonin, 1999; Archibugi y Pietrobelli, 2003). El conocimiento tácito de mercadotecnia es significativo porque actúa como un proceso social por el cual los empleados con conocimientos en la disciplina adquieren y transfieren a otros sus aprendizajes (Ambrosini y Bowman, 2001). Este conocimiento suele estar enraizado contextual, organizacional y socialmente dentro de las organizaciones (Inkpen, 2008), pero sumando a ello las características del sector hotelero (Hjalager, 2002), se vuelve relevante propiciar mecanismos de transferencia, a fin de aprovecharlo en diversos aspectos mercadológicos, como por ejemplo, innovación y desarrollo de nuevos productos (Huang, Chang, y Henderson, 2008), confianza y la colaboración (Szulanski, 2000), mercadotecnia relacional (Gummesson, 2017a) y fortalecimiento de una ventaja competitiva.

La adquisición de conocimientos puede tener múltiples antecedentes (Liao y Barnes, 2015), no obstante, en esta investigación se estudia la capacidad de absorción de conocimiento en la disciplina, la cultura abierta de colaboración, la confianza entre compañeros de trabajo y la riqueza de medios de comunicación como variables de impacto relevante en adquisición de conocimiento de mercadotecnia y el efecto de ésta en la transferencia.

Capacidad de absorción del conocimiento de mercadotecnia y adquisición de conocimiento tácito de mercadotecnia

La capacidad de absorción se refiere a la habilidad de absorber conocimientos esperando con ello, que se adquieran nuevos conocimientos y luego puedan ser compartidos (Grant, 1996). Esta capacidad se identifica con el conjunto de procesos y rutinas organizacionales que permiten identificar, asimilar, transformar y explotar nuevos conocimientos del ambiente (Cohen y Levinthal, 1989; Zahra y George, 2002), por tanto, requiere del consentimiento para el intercambio de ideas y permitir que las empresas sean más comprometidas en la adquisición de aquél (Yli-Renko, Autio, y Sapienza, 2001). 
Rodríguez Antón, Oliva, y Laguna (2003) mencionan que la situación hotelera actual es de estancamiento de la demanda, cambio continuo y necesidad de aprendizaje constante, sin embargo, puede ser difícil de evaluar el efecto de la capacidad de absorción (Berchicci, 2013) y específicamente en la evaluación de los efectos de ésta sobre el conocimiento tácito aún prevalece la escasez de estudios empíricos (Jafari y Mozhden, 2013). La capacidad de absorción combina el conocimiento existente con el nuevo (Lane, Koka, y Pathak, 2006), de tal forma que impacta en el desarrollo del conocimiento y el potencial de una firma para innovar y mejorar su desempeño competitivo (Zahra y George, 2002).

La adquisición de conocimiento no puede entenderse sin la capacidad de absorción ya que potencialmente ese nuevo conocimiento puede ser asimilado, transformado y explotado (Lane et al., 2006; Johnson, 2017). En el campo de las cadenas hoteleras se presentan altas tasas de rotación de empleados y con seguridad ello afecta la retención de la información y del conocimiento existente en la organización. El contacto del personal con los clientes es fuente de información fundamental sobre los mercados y el propio negocio. Por lo tanto, se presenta una causa para que la adquisición y la gestión del conocimiento sean implementadas de manera profesional en el sector hotelero (Gjelsvik, 2002; Yang y Wan, 2004). Por esta razón es posible suponer que:

H1: En los pequeños y medianos hoteles de Playa del Carmen, la capacidad de absorción de conocimientos de mercadotecnia tiene un efecto positivo en la adquisición de conocimiento tácito de mercadotecnia.

Cultura abierta de colaboración entre compañeros y su efecto en la adquisición de conocimiento tácito de mercadotecnia

Al interior de las instituciones se observa la necesidad de contar con una cultura de colaboración que motive a los integrantes de la organización a buscar nuevas formas de implementar procesos, donde los horarios de trabajo y la interacción entre sus miembros contribuyan a adquirir y compartir conocimiento (Kucharska y Kowalczyk, 2016).

La cultura de colaboración es una de las condiciones más importantes que pueden conducir al éxito de un negocio. La mayoría de los empleados tienen una curiosidad y disposición intelectual que puede facilitar la adquisición de conocimiento y, por tanto, compartirlo (Davenport y Prusak, 2001; Kucharska y Kowalczyk, 2016). En la generación del conocimiento intraorganizacional se requiere de una comunicación intensiva, una cultura que acepte nuevas ideas y esté preparada para apoyar la exploración de sus procesos y actividades (Ruggles, 1998).

Algunas investigaciones (Zapata, 2004) han encontrado que la cultura organizativa es el principal facilitador del conocimiento y que culturas más abiertas y orientadas al cambio están mejor capacitadas para adquirir y transferir el conocimiento internamente. Investigaciones en diferentes ámbitos han encontrado que la cultura de la organización tiene un efecto positivo sobre la adquisición de conocimiento (Liao, Chang, Hu, y Yueh, 2012). Se ha encontrado que una firma con una cultura abierta y flexible es más propensa a comprometerse con las acciones de aprendizaje, la modificación de rutinas, procesos o de la cadena de valor en favor de una dirección estratégica adecuada. Por tanto, en función de lo anteriormente expuesto se plantea que: 
H2. En los pequeños y medianos hoteles de Playa del Carmen, una cultura abierta de colaboración entre compañeros de trabajo influye de manera positiva en la adquisición de conocimiento tácito.

\section{Confianza mutua entre compañeros y adquisición de conocimiento tácito de mercadotecnia}

La confianza mutua se refiere a la creencia o expectativa de que para llevar a cabo ciertas actividades se puede solicitar el apoyo a otro compañero con la certeza de que va a corresponder con la responsabilidad necesaria (Young y Wilkinson, 1989), y que la otra persona no se aprovechará de las acciones de vulnerabilidad en la que se encuentra el solicitante (Swan, Trawick, Rink, y Roberts, 1988). La confianza es un factor importante en la construcción de relaciones sociales y existe cuando una de las partes cree en la fiabilidad e integridad del otro (Morgan y Hunt, 1994; Hunt. Arnett, y Madhavaram, 2006) y descansa en las expectativas y predicciones de colaboración entre las partes involucradas (Maurer, 2010).

La confianza actúa en las relaciones y facilita tanto el acceso como el intercambio de conocimientos (Arrow, 1974; Nahapiet y Ghoshal, 1998). Es importante en todo tipo de intercambio social, incluyendo las relaciones interinstitucionales e intraorganizacionales (Massey y Dawes, 2007). Además, desempeña un papel central en los procesos de adquisición e intercambio de conocimientos (Dhanaraj, Lyles, Steensma, y Tihanyi, 2004; Maurer, 2010).

Investigaciones anteriores han encontrado que la confianza genera beneficios a la organización a través de la interacción y la colaboración (Dirks y Ferrin, 2001), además, se ha evidenciado que la confianza incrementa la adquisición de conocimiento entre los miembros de una firma o entre aliados, lo que conlleva a nuevas oportunidades de negocio (Tsai y Ghohal, 1998; Maurer, 2010). En el ámbito de la mercadotecnia, Arnett y Wittmann (2014) probaron un modelo de intercambio de conocimiento tácito entre las ventas y la mercadotecnia y concluyeron que cuando la confianza en el compañero de trabajo es mayor, el intercambio de conocimiento tácito tiende a incrementarse.

Por su propia naturaleza, el conocimiento tácito requiere interacciones personales frecuentes para la adquisición y la transferencia de conocimiento tácito con los demás miembros de la organización. Así, las relaciones que se caracterizan por tener altos niveles de confianza son más propicias para incentivar la adquisición (Maurer, 2010) y la transferencia de conocimiento tácito (Lin, 2007). Por lo anterior, es posible suponer que:

H3. La Confianza en compañeros de trabajo tiene un efecto positivo en la adquisición de conocimiento tácito de mercadotecnia.

Riqueza de medios de comunicación y adquisición de conocimientos tácitos de mercadotecnia

Los procesos de comunicación pueden convertirse en conductores o barreras para el desarrollo de la voluntad a compartir el conocimiento (Wiewiora, Trigunarsyah, Murphy, y Coffey, 2013), pero también funciona como un mecanismo relevante para la adquisición de conocimiento de fuentes internas y externas (Boiral, 2002), así como la asimilación de prácticas en las pequeñas y medianas empresas (Johnson, 2017).

Varios autores (Mirc, 2013; Wiewiora et al., 2013) están de acuerdo en que la integración de equipos y reuniones regulares son esenciales para el proceso de adquisición y transferencia 
de conocimientos y se considera que la aplicación de las rotaciones de trabajo, equipos de integración transversal de puestos de trabajo y una comunicación regular facilitan que los conocimientos fluyan entre los miembros de una organización (Mirc, 2013).

Aunque existen varios mecanismos de comunicación, también la comunicación informal intraorganizacional ofrece oportunidades para la adquisición y transferencia del conocimiento tácito en la organización (Dyer y Hatch, 2006), la interacción y las comunidades de práctica entre los miembros de la firma pueden convertirse en conductores de este proceso porque brindan la oportunidad de compartir conocimiento, incluso por iniciativa de los propios colaboradores (Zapata, 2004). Por tanto, se propone que:

H4. En los pequeños y medianos hoteles de Playa del Carmen, la riqueza de medios de comunicación influye de manera positiva en la adquisición de conocimiento tácito de mercadotecnia entre los empleados.

\section{Adquisición y transferencia de conocimientos tácitos de mercadotecnia}

El proceso de adquisición y transferencia de conocimientos sin duda implica a varias personas clave, una de las cuales es el titular del conocimiento y otro es el receptor del conocimiento. El resultado de la adquisición de conocimientos depende del esfuerzo de ambas partes. Por tanto, para motivar la adquisición, el contenido de los conocimientos de un receptor y la fuente deben percibir el conocimiento como valiosos (Desouza, Awazu, y Wan, 2006).

La adquisición de conocimiento tácito requiere contacto continuo e intenso entre los empleados (Kale, Singh, y Perlmutter, 2000) y cuando se transfiere el conocimiento de una persona a otra, el conocimiento es interpretado por el receptor en base a lo que sabe y su experiencia. Lo cual puede tener diferente significado y valor en el nuevo entorno. En el campo de la mercadotecnia se ha encontrado que el conocimiento tácito es un recurso valioso, que, al compartirse incrementa las oportunidades de desarrollo de nuevas estrategias en las distintas áreas de la mercadotecnia. El bloqueo y las barreras organizacionales para su desarrollo pueden dar resultados sólo moderados (Amiryany, Ard/Pieter, y Cloodt, 2012; Harvey, 2012). Por tanto, se plantea que:

H5. La adquisición de conocimientos tácitos de mercadotecnia influye positivamente sobre la transferencia de conocimientos tácitos de mercadotecnia.

En la figura 1 se presenta el modelo conceptual de transferencia de conocimiento tácito que esta investigación plantea. Este modelo representa los vínculos relacionales entre las construcciones que representa Capacidad de Absorción de Conocimiento de Mercadotecnia (CACM), Cultura Abierta de Colaboración (CAC), Confianza en Compañeros de Trabajo (CCT) y Riqueza de Medios de Comunicación (RMC), la Adquisición de Conocimiento Tácito de Mercadotecnia (ACTM), y a su vez con la transferencia de conocimiento tácito de mercadotecnia (TCTM). También indica la relación hipotética entre las variables. 


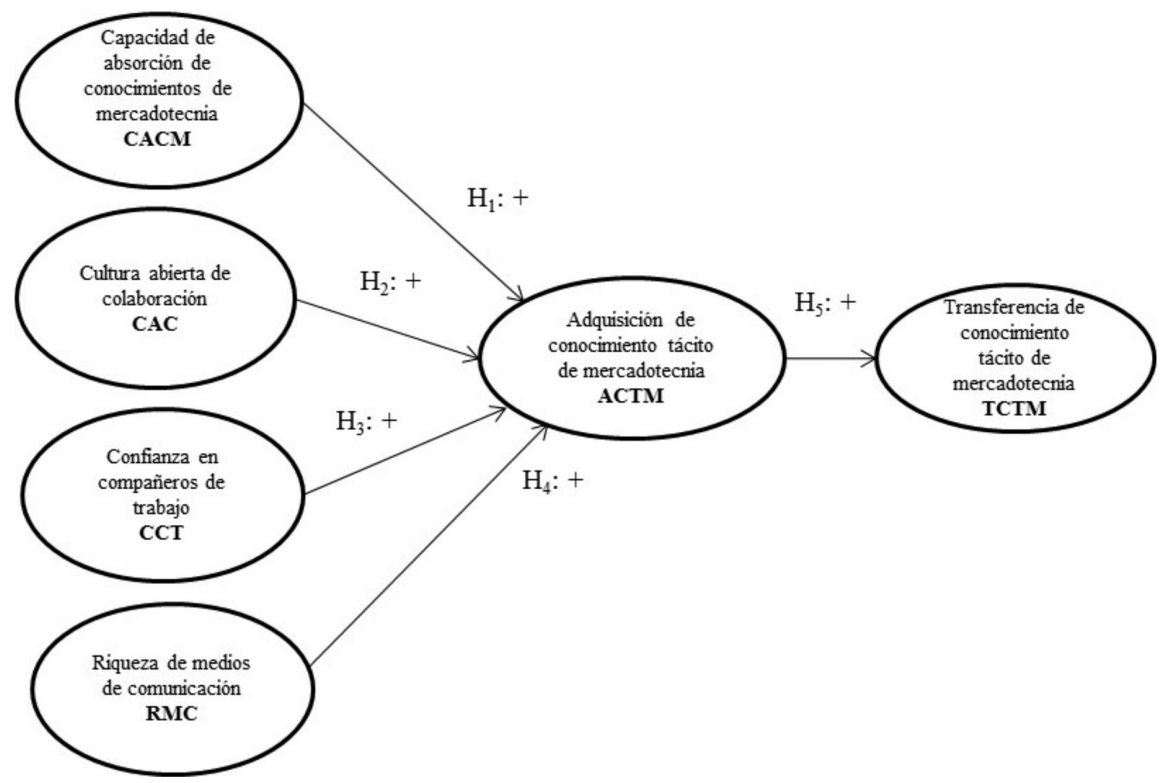

Figura 1. Modelo conceptual de los antecedentes de la adquisición del conocimiento tácito de mercadotecnia y el impacto de ésta sobre la transferencia de conocimiento Fuente: Elaboración propia.

\section{Metodología}

Se desarrolló una investigación cuantitativa y transversal, ya que se buscó obtener información para probar las hipótesis de las relaciones establecidas entre las variables del estudio (Hair, Bush, y Ortinau, 2010). La recolección de datos fue a través de encuestas personales administradas cara a cara a gerentes o administradores, jefes de recepción, ventas, servicio al cliente y servicio a habitación, cuyas funciones se vinculaban con tareas relacionadas con mercadotecnia en hoteles de tamaño pequeño y mediano en la ciudad de Playa del Carmen, en Quintana Roo, México.

Se obtuvieron en total 229 encuestas utilizando una técnica de muestreo no probabilístico por conveniencia para seleccionar a los encuestados potenciales, durante los meses de octubre a diciembre de 2015. La unidad de muestreo fueron 141 hoteles ubicados en la Ciudad de Playa del Carmen, Quintana Roo, lográndose una cobertura del 81.9\% de los establecimientos reportados por el Fideicomiso de Promoción Turística Riviera Maya (2015). Se contó con una participación en el estudio de $45 \%$ mujeres y $55 \%$ de hombres, de los cuales el $43 \%$ tenía un tiempo de permanencia en el hotel de hasta un año y el 37.6\% señaló contar con una permanencia entre 2 y 5 años. La mayor parte de los hoteles seleccionados consideraron tener una categoría de 2 y 3 estrellas (19.9\% y $49.6 \%$ respectivamente). Los hoteles de una estrella representaron el $16.3 \%$ de la muestra y los de 4 estrellas conformaron el 14.2\%. Respecto al nivel educativo del personal encuestado (Anexo 1) poco más de la mitad (55\%) contaba con estudios de bachillerato y alrededor del $40 \%$ con estudios de licenciatura. 


\section{Medición}

El instrumento utilizado comprende 29 ítems. Las escalas de medición son de intervalo a través de escalas de Likert de 5 y 7 puntos (Hair et al., 2010). El alfa de Cronbach -conjunta- de las variables medidas fue de 0.819 .

En el análisis de las dimensiones se encontró que la desviación estándar en todos los ítems es superior a 1; por otro lado, en el alpha de Cronbach donde se evaluó la consistencia interna, el primer constructo "capacidad de absorción de conocimientos de mercadotecnia" arrojó un alpha de 0. 932. Garson (2010) comenta que en ciencias sociales el corte del alpha debe ser 0.80 o superior para un conjunto de elementos a tener en cuenta para una escala, pero es posible utilizar 0.70 como aceptable para una investigación confirmatoria (Cronbach, 1951, Thiétart, 2001). En el segundo constructo, "cultura abierta a la colaboración”, arrojó un alpha de 0.865, en el tercer constructo "Confianza en compañeros de trabajo", dio un alpha de 0.919, en el cuarto constructo "distancia cultural", arrojó el alpha de 0.779 , siendo ésta consistencia la más baja de todos por lo que se tomó la decisión de eliminarlo de la evaluación del modelo. El valor más bajo de todos, en el quinto constructo "riqueza de medios de comunicación" 0.816, en el sexto constructo "adquisición de conocimiento tácito de mercadotecnia", arrojó un alpha de 0.943 y en el séptimo y último constructo "transferencia de conocimiento tácito de mercadotecnia", arrojó un alpha de 0.874 .

\section{Análisis de resultados}

\section{Modelo de medición}

Para el análisis estadístico de los datos se siguió el procedimiento de la técnica de modelos de ecuaciones estructurales (MEC) para examinar modelos complejos que presentan un gran número de constructos, indicadores y relaciones (Barclay et al., 1995). Debido a que Partial Least Squares (PLS) permite trabajar con muestras pequeñas y posee supuestos menos estrictos respecto a la distribución de los datos (Chin y Newsted, 1999). En Partial Least Squares Structural Equation Modeling (PLS-SEM), el modelo es descrito por dos componentes (Tenenhaus et al., 2005): 1) el modelo de medición, el cual relaciona las variables manifiestas con la variable latente y 2) el modelo estructural, el cual muestra la relación entre las variables latentes. Los modelos estructurales se validan en dos etapas (Henseler, Ringle, y Sinkovicks, 2009): 1) revisión del modelo de medición a través de diversos procedimientos y 2) validación del modelo estructural.

En base a la regla propuesta por Hulland (1999) sobre conservar los ítems con cargas de 0.7 o más, aquellos indicadores de los modelos de medición reflectivos que no alcanzaron el nivel aceptable de fiabilidad (anexo 2) fueron eliminados. La validez de convergencia es evaluada con el valor del Promedio de Varianza Extraída (Average Variance Extracted, AVE por sus siglas en inglés), el cual debe ser mayor a 0.5 según el criterio de Fornell-Larcker (Seidel y Back, 2009). En el modelo final (modelo 2), los constructos reflectivos presentan un valor mínimo por arriba del límite (0.59) La validez de convergencia también es demostrada cuando los ítems cargan de manera alta en sus factores asociados (carga mayor a 0.5) y éstas deben ser mayores en el constructo asignado que en cualquier otro. Las cargas cruzadas de los indicadores reflectivos muestran una carga mayor en valor absoluto en el constructo al que 
han sido asignados (anexo 3), en relación a cualquier otro constructo reflectivo (Seidel y Back, 2009).

El coeficiente Rho de Dillon-Goldsteins (índice de fiabilidad compuesta) es utilizado para evaluar la consistencia interna (Fornell y Larcker, 1981). En el modelo, el índice de fiabilidad compuesta para los constructos reflectivos, fue mayor a 0.88 (anexo 4), excediendo el valor mínimo aceptable de 0.70 (Seidel y Back, 2009; Hair, Anderson, Tatham, y Black, 1998). En cuanto a la validez discriminante, Fornell y Larcker (1981) sugieren que un puntaje de 0.5 del AVE, indica un nivel aceptable de validez discriminante. El promedio de varianza extraída para los constructos reflectivos varía entre .59 y .87 (anexo 2). Tal validez, para los modelos de medición reflectivos también es demostrada cuando la raíz del promedio de AVE de cada constructo es mayor que la correlación con cualquier otra variable latente (Seidel y Back, 2009; Delić y Lenz, 2008). En el anexo 4, se presenta la matriz que compara la raíz de AVE en la diagonal de las tablas con el triángulo superior de la matriz que contiene las correlaciones entre constructos. La raíz AVE es más grande que la correlación para cada columna y fila respectiva de los constructos reflectivos, sugiriendo validez discriminante (Seidel y Back, 2009; Duarte y Raposo, 2010) y convergente aceptable (Chin, 1998; Duarte y Raposo, 2010). Seidel y Back (2009), coinciden respecto a que la validez discriminante se cumple al comparar las cargas cruzadas de los indicadores asignados al constructo reflectivo, contra el resto de ellas. En el modelo, no se revelan problemas de validez discriminante (anexo 5), dado que todos los indicadores muestran cargas mayores en su respectivo constructo con respecto a otros constructos reflectivos (Duarte y Raposo, 2010).

\section{Modelo estructural}

El modelo explica el $26.80 \%$ de la varianza de TCTM $\left(\mathrm{R}^{2}=26.80\right)$, directamente a través de ACTM e indirectamente por CCT y RMC. La técnica de bootstrap con 200 submuestras fue empleada para estimar la significancia de los coeficientes path en el modelo y comparar los estimadores estadísticamente. Los resultados del modelo propuesto (figura 2) respaldan el 60\% de nuestras hipótesis (tabla 1), con un nivel de confianza del 95\%.

Tabla 1

Evaluación de los efectos en el modelo 2

\begin{tabular}{cccccc}
\hline Hipótesis & Path & $\begin{array}{c}\text { Coeficiente } \\
\text { Path }\end{array}$ & $\begin{array}{c}\text { Percentil } \\
\mathbf{0 . 0 2 5} *\end{array}$ & $\begin{array}{c}\text { Percentil } \\
\mathbf{0 . 9 7 5}^{*}\end{array}$ & $\begin{array}{c}\text { Cumplimiento } \\
\text { de hipótesis }\end{array}$ \\
\hline H1 & CACM -> ACTM & 0.010 & -0.105 & 0.128 & No rechazo \\
H2 & CAC - > ACTM & 0.084 & -0.075 & 0.255 & No rechazo \\
H3 & CCT -> ACTM & 0.362 & 0.208 & 0.486 & Rechazo \\
H4 & RMC ->ACTM & 0.377 & 0.193 & 0.538 & Rechazo \\
H5 & ACTM-> TCTM & 0.518 & 0.413 & 0.610 & Rechazo \\
\hline
\end{tabular}

*significativo con alpha de 0.05 , para una prueba de dos colas, Ho: el coeficiente path es igual a 0. 
Los resultados en la figura 2 indicaron un efecto directo, positivo y significativo de ACTM en TCTM $\left(\beta_{5}=0.5177\right)$. Adicionalmente, el efecto indirecto de $\operatorname{CCT}$ en $\operatorname{TCTM}\left(\beta_{3^{*}} \beta_{5}\right)$, resultado de aplicar la técnica de bootstraping (Kenny, 2015) fue significativo de 0.1848 (IC 95\%: .1089-.2617). Similarmente, RMC apoyó indirectamente a TCTM, con un efecto de .1932 (IC 95\%: .0975-.2829).

La hipótesis planteada respecto a RMC y ACTM, resultó significativa y en la dirección esperada. Tal que RMC incide directa y positivamente sobre ACTM (H4: $\left.\beta_{4}=0.3769\right)$. En relación a CCT, el modelo indica la fuerza de la relación directa y significativa con ACTM $\left(\beta_{3}=-0.3622\right)$, apoyando la hipótesis H3.



Figura 2. Modelo final de TCTM con coeficientes path significativos

\section{Discusión y conclusiones}

En la prueba empírica se encontró que la confianza en compañeros de trabajo y la riqueza de medios de comunicación, afectan de manera positiva en la adquisición de conocimiento tácito de mercadotecnia en los pequeños y medianos hoteles de la ciudad de Playa del Carmen, mientras que la capacidad de absorción de conocimiento de mercadotecnia y la cultura abierta de colaboración no tienen una tienen un efecto significativo para la adquisición de dicho conocimiento.

A diferencia de lo planteado, la hipótesis 1 no pudo aceptarse ya que la capacidad de absorción de conocimientos de mercadotecnia no tuvo un efecto significativo en la adquisición de conocimiento tácito de mercadotecnia. La teoría vincula estrechamente la capacidad de absorción con la adquisición de conocimiento, especialmente con los aspectos de identificación 
y asimilación de nuevos conocimientos (Johnson, 2017). Dados los resultados en el sector y el contexto estudiado, consideramos que es necesario seguir estudiando el comportamiento de este efecto en el sector hotelero en México y particularmente en las pequeñas y medianas empresas, ya que la capacidad de absorción se manifiesta como una habilidad y anhelo de la necesidad de aprendizaje (Rodríguez et al., 2003) y el aprovechamiento del conocimiento para mejorar el desempeño personal y organizacional.

Se encontró que una cultura abierta de colaboración entre compañeros no tiene un efecto en la adquisición de conocimiento tácito de mercadotecnia, por lo que la hipótesis 2 no pudo ser verificada. Investigaciones empíricas (Zapata, 2004) han encontrado que la cultura es un factor relevante (Liao et al., 2012), incluso, el más importante predictor del conocimiento. Sin embargo, entre los colaboradores de mercadotecnia de los hoteles de Playa del Carmen, una cultura abierta de colaboración no afecta a la adquisición de conocimiento. Una posible explicación de esto podría deberse a una mayor necesidad, por parte de las empresas hoteleras estudiadas, a desarrollar los factores de contingencia de la cultura organizacional sustentados en la selección, capacitación, liderazgo y su proceso de profesionalización del factor humano (Flores-Ortiz, Vega-López, y Chávez-Moreno, 2015). Dado que una cultura organizacional abierta y flexible se encuentra permanentemente en la posibilidad de aprender y compartir conocimientos, es recomendable seguir estudiando el comportamiento de esta variable en hoteles pequeños y medianos en otros contextos.

A diferencia de lo anterior, se encontró que la confianza en los compañeros de trabajo afecta la adquisición de conocimiento táctico de mercadotecnia, por lo que se comprobó la hipótesis tres. La confianza se basa en aspectos positivos que contribuyen en la posibilidad de adquirir y transferir conocimientos que apoyen una construcción de acciones organizacionales propositivas basadas en la colaboración (Maurer, 2010). Por tanto, la pequeña y mediana empresa de la ciudad de Playa del Carmen debe estimular las interacciones entre los miembros de la organización para que los conocimientos basados en la experiencia y juicio mercadológico se conviertan en una dinámica y práctica común en la organización.

Además, se comprobó la hipótesis cuatro, ya que la riqueza de los medios de comunicación de la organización tiene un efecto importante y significativo sobre la adquisición de conocimiento tácito de mercadotecnia. Los medios de comunicación utilizados por estas empresas parecen facilitar la adquisición de conocimiento tácito con rapidez y eficiencia (Zapata, 2004). Los medios de comunicación utilizados pueden incluir reuniones, opiniones, sugerencias, correos electrónicos y cualquier otro medio que facilite que los empleados del área de mercadotecnia de los hoteles adquieran nuevos conocimientos tácitos para el desempeño de sus tareas. Finalmente, la capitalización de la adquisición del conocimiento tácito de mercadotecnia se da cuando los receptores además de adoptar, aplican el nuevo conocimiento en las actividades que desarrollan en sus puestos de trabajo, esto hace que el conocimiento sea significativo (Zapata, 2004), pero también propicia que pueda transferirse a otros, lo que se convierte en un proceso valioso para la organización, ya que el conocimiento tácito, por su naturaleza, suele ser más personal y difícil de comunicarse o transferirse.

Este estudio realiza una pequeña contribución a la comprensión de cómo se relacionan variables clave en la adquisición de conocimiento tácito de mercadotecnia y corrobora el efecto de ésta sobre transferencia de conocimiento de mercadotecnia en las pequeñas y medianas empresas de alojamiento turístico en un contexto mexicano. Sin embargo, la investigación presenta ciertas limitaciones, ya que los datos de la investigación fueron recolectados siguiendo 
un muestreo no probabilístico de conveniencia. Por tanto, los hallazgos del presente estudio no son generalizables a la población, ni pueden extenderse al sector hotelero mexicano. Por tanto, para estudios futuros, podría ser relevante replicar la investigación en otras regiones de México, otros tamaños de hoteles, o bien en empresas vinculadas al sector (restaurantes, comercios, entre otros). Además, también se sugiere evaluar el impacto de la transferencia en otras variables como rentabilidad, utilidades, ventaja competitiva y posicionamiento de marca.

Anexo 1. Datos eliminar de los encuestados.

\begin{tabular}{|c|c|c|c|c|}
\hline & Frecuencia & Porcentaje & $\begin{array}{c}\text { Porcentaje } \\
\text { válido }\end{array}$ & $\begin{array}{l}\text { Porcentaje } \\
\text { acumulado }\end{array}$ \\
\hline \multicolumn{5}{|c|}{ Género } \\
\hline Hombre & 126 & 55.0 & 55.0 & 55.0 \\
\hline Mujer & 103 & 45.0 & 45.0 & 100.0 \\
\hline Total & 229 & 100.0 & 100.0 & \\
\hline \multicolumn{5}{|c|}{ Permanencia en la industria hotelera en años } \\
\hline Hasta un año & 99 & 43.2 & 43.2 & 43.2 \\
\hline de 2 a 5 & 86 & 37.6 & 37.6 & 80.8 \\
\hline De 6 a 9 & 28 & 12.2 & 12.2 & 93.0 \\
\hline De 10 a 13 & 10 & 4.4 & 4.4 & 97.4 \\
\hline De 14 a 17 & 5 & 2.2 & 2.2 & 99.6 \\
\hline De 18 a 21 & 1 & .4 & .4 & 100.0 \\
\hline Total & 229 & 100.0 & 100.0 & \\
\hline \multicolumn{5}{|c|}{ Tipo de hotel } \\
\hline Una estrella & 23 & 16.3 & 16.3 & 16.3 \\
\hline Dos estrellas & 28 & 19.9 & 19.9 & 36.2 \\
\hline Tres estrellas & 70 & 49.6 & 49.6 & 85.8 \\
\hline Cuatro estrellas & 20 & 14.2 & 14.2 & 100 \\
\hline Total & 141 & 100.0 & 100.0 & \\
\hline \multicolumn{5}{|c|}{ Nivel educativo } \\
\hline Secundaria & 7 & 3.1 & 3.1 & 3.1 \\
\hline Bachillerato & 126 & 55.0 & 55.0 & 58.1 \\
\hline Licenciatura & 91 & 39.7 & 39.7 & 97.8 \\
\hline Maestría & 5 & 2.2 & 2.2 & 100.0 \\
\hline Total & 229 & 100.0 & 100.0 & \\
\hline \multicolumn{5}{|c|}{ Departamento en el que trabaja } \\
\hline Servicio al cliente & 29 & 12.7 & 1.7 & 1.7 \\
\hline Ventas & 120 & 52.4 & 52.4 & 54.1 \\
\hline Mercadotecnia y Finanzas & 7 & 3.1 & 3.1 & 57.2 \\
\hline Gerencia & 42 & 18.3 & 18.3 & 75.5 \\
\hline Jefe de Recepción & 27 & 11.8 & 22.7 & 98.3 \\
\hline Servicio habitación & 4 & 1.7 & 1.7 & 100.0 \\
\hline Total & 229 & 100.0 & 100.0 & \\
\hline
\end{tabular}

*significativo con alpha de 0.05 , para una prueba de dos colas, Ho: el coeficiente path es igual a 0. 
http://dx.doi.org/10.22201/fca.24488410e.2018.1364

Anexo 2. Cargas de los indicadores reflectivos $(n=229)$.

\begin{tabular}{|c|c|c|c|c|}
\hline & Frequency & Percentage & $\begin{array}{c}\text { Valid } \\
\text { percentage }\end{array}$ & $\begin{array}{c}\text { Accumulated } \\
\text { percentage }\end{array}$ \\
\hline Man & 126 & 55.0 & 55.0 & 55.0 \\
\hline Woman & 103 & 45.0 & 45.0 & 100.0 \\
\hline Total & 229 & 100.0 & 100.0 & \\
\hline \multicolumn{5}{|c|}{ Permanence in the hotel industry in years } \\
\hline Up to one year & 99 & 43.2 & 43.2 & 43.2 \\
\hline from 2 to 5 & 86 & 37.6 & 37.6 & 80.8 \\
\hline from 6 to 9 & 28 & 12.2 & 12.2 & 93.0 \\
\hline from 10 to 13 & 10 & 4.4 & 4.4 & 97.4 \\
\hline from 14 to 17 & 5 & 2.2 & 2.2 & 99.6 \\
\hline from 18 to 21 & 1 & .4 & .4 & 100.0 \\
\hline Total & 229 & 100.0 & 100.0 & \\
\hline \multicolumn{5}{|c|}{ Type of hotel } \\
\hline One star & 23 & 16.3 & 16.3 & 16.3 \\
\hline Two stars & 28 & 19.9 & 19.9 & 36.2 \\
\hline Three stars & 70 & 49.6 & 49.6 & 85.8 \\
\hline Four stars & 20 & 14.2 & 14.2 & 100 \\
\hline Total & 141 & 100.0 & 100.0 & \\
\hline \multicolumn{5}{|c|}{ Level of education } \\
\hline Middle school & 7 & 3.1 & 3.1 & 3.1 \\
\hline High school & 126 & 55.0 & 55.0 & 58.1 \\
\hline Bachelor degree & 91 & 39.7 & 39.7 & 97.8 \\
\hline Master's degree & 5 & 2.2 & 2.2 & 100.0 \\
\hline Total & 229 & 100.0 & 100.0 & \\
\hline \multicolumn{5}{|c|}{ Department in which they work } \\
\hline Customer service & 29 & 12.7 & 1.7 & 1.7 \\
\hline Sales & 120 & 52.4 & 52.4 & 54.1 \\
\hline Marketing and finances & 7 & 3.1 & 3.1 & 57.2 \\
\hline Management & 42 & 18.3 & 18.3 & 75.5 \\
\hline Reception manager & 27 & 11.8 & 22.7 & 98.3 \\
\hline Room service & 4 & 1.7 & 1.7 & 100.0 \\
\hline Total & 229 & 100.0 & 100.0 & \\
\hline
\end{tabular}

*Significant with an alpha of 0.05 , for a two-tailed test, ho: the path coefficient is equal to 0 . 
Annex 3. Cross-loads of the reflective indicators of Model $2(n=229)$.

\begin{tabular}{ccccccc}
\hline & \multicolumn{7}{c}{ Constructo } \\
\cline { 2 - 7 } ítem & CACM & CAC & CCT & RMC & ACTM & TCTM \\
\hline BC1 & 0.917 & 0.543 & 0.632 & 0.287 & 0.390 & 0.368 \\
BC2 & 0.919 & 0.498 & 0.603 & 0.197 & 0.321 & 0.319 \\
BC3 & 0.924 & 0.506 & 0.580 & 0.200 & 0.302 & 0.323 \\
BC4 & 0.894 & 0.496 & 0.530 & 0.209 & 0.370 & 0.309 \\
NO5 & 0.548 & 0.739 & 0.471 & 0.362 & 0.409 & 0.372 \\
NO6 & 0.319 & 0.732 & 0.379 & 0.405 & 0.306 & 0.325 \\
NO7 & 0.479 & 0.766 & 0.435 & 0.405 & 0.467 & 0.389 \\
NO8 & 0.431 & 0.803 & 0.523 & 0.436 & 0.426 & 0.442 \\
NO9 & 0.391 & 0.789 & 0.364 & 0.442 & 0.294 & 0.385 \\
NO10 & 0.368 & 0.791 & 0.432 & 0.462 & 0.349 & 0.444 \\
CC11 & 0.611 & 0.567 & 0.890 & 0.392 & 0.472 & 0.405 \\
CC12 & 0.575 & 0.514 & 0.949 & 0.407 & 0.562 & 0.404 \\
CC13 & 0.607 & 0.520 & 0.943 & 0.372 & 0.559 & 0.355 \\
RMC19 & 0.224 & 0.455 & 0.329 & 0.794 & 0.441 & 0.343 \\
RMC20 & 0.151 & 0.483 & 0.362 & 0.829 & 0.412 & 0.464 \\
RMC21 & 0.236 & 0.447 & 0.378 & 0.864 & 0.563 & 0.445 \\
RMC22 & 0.173 & 0.354 & 0.271 & 0.718 & 0.410 & 0.285 \\
TCT23 & 0.383 & 0.497 & 0.583 & 0.591 & 0.934 & 0.475 \\
TCT24 & 0.348 & 0.470 & 0.578 & 0.550 & 0.944 & 0.478 \\
TCT25 & 0.290 & 0.366 & 0.351 & 0.383 & 0.800 & 0.441 \\
ACT26 & 0.343 & 0.432 & 0.378 & 0.430 & 0.476 & 0.905 \\
ACT27 & 0.370 & 0.537 & 0.384 & 0.467 & 0.471 & 0.924 \\
ACT28 & 0.315 & 0.447 & 0.403 & 0.462 & 0.523 & 0.941 \\
ACT29 & 0.314 & 0.486 & 0.372 & 0.422 & 0.436 & 0.928 \\
\hline
\end{tabular}

Annex 4. Correlation matrix between constructs and AVE roots greater than the correlations and Dillon-Goldstein. Rho. Index for Model 2 ( $\mathrm{n}=229)$.

\begin{tabular}{|c|c|c|c|c|c|c|}
\hline & CACM & $\mathrm{CAC}$ & $\mathrm{CCT}$ & $\mathrm{RMC}$ & АCTM TCTM & $\begin{array}{l}\text { Rho de Dillon- } \\
\text { Goldsteins }\end{array}$ \\
\hline CACM & $\underline{0.91}$ & & & & & 0.95 \\
\hline $\mathrm{CAC}$ & 0.57 & $\underline{0.77}$ & & & & 0.90 \\
\hline $\mathrm{CCT}$ & 0.67 & 0.59 & $\underline{0.93}$ & & & 0.95 \\
\hline $\mathrm{RMC}$ & 0.26 & 0.64 & 0.47 & $\underline{0.80}$ & & 0.88 \\
\hline ACTM & 0.39 & 0.52 & 0.65 & 0.65 & $\underline{0.90}$ & 0.92 \\
\hline TCTM & 0.38 & 0.56 & 0.43 & 0.55 & $\underline{0.92}$ & 0.96 \\
\hline
\end{tabular}


Annex 5. Cross-loads of the reflective indicators of Model $2(n=229)$.

\begin{tabular}{ccccccc}
\hline & \multicolumn{7}{c}{ Constructo } \\
\cline { 2 - 7 } ítem & CACM & CAC & CCT & RMC & ACTM & TCTM \\
\hline BC1 & 0.917 & 0.543 & 0.632 & 0.287 & 0.390 & 0.368 \\
BC2 & 0.919 & 0.498 & 0.603 & 0.197 & 0.321 & 0.319 \\
BC3 & 0.924 & 0.506 & 0.580 & 0.200 & 0.302 & 0.323 \\
BC4 & 0.894 & 0.496 & 0.530 & 0.209 & 0.370 & 0.309 \\
NO5 & 0.548 & 0.739 & 0.471 & 0.362 & 0.409 & 0.372 \\
NO6 & 0.319 & 0.732 & 0.379 & 0.405 & 0.306 & 0.325 \\
NO7 & 0.479 & 0.766 & 0.435 & 0.405 & 0.467 & 0.389 \\
NO8 & 0.431 & 0.803 & 0.523 & 0.436 & 0.426 & 0.442 \\
NO9 & 0.391 & 0.789 & 0.364 & 0.442 & 0.294 & 0.385 \\
NO10 & 0.368 & 0.791 & 0.432 & 0.462 & 0.349 & 0.444 \\
CC11 & 0.611 & 0.567 & 0.890 & 0.392 & 0.472 & 0.405 \\
CC12 & 0.575 & 0.514 & 0.949 & 0.407 & 0.562 & 0.404 \\
CC13 & 0.607 & 0.520 & 0.943 & 0.372 & 0.559 & 0.355 \\
RMC19 & 0.224 & 0.455 & 0.329 & 0.794 & 0.441 & 0.343 \\
RMC20 & 0.151 & 0.483 & 0.362 & 0.829 & 0.412 & 0.464 \\
RMC21 & 0.236 & 0.447 & 0.378 & 0.864 & 0.563 & 0.445 \\
RMC22 & 0.173 & 0.354 & 0.271 & 0.718 & 0.410 & 0.285 \\
TCT23 & 0.383 & 0.497 & 0.583 & 0.591 & 0.934 & 0.475 \\
TCT24 & 0.348 & 0.470 & 0.578 & 0.550 & 0.944 & 0.478 \\
TCT25 & 0.290 & 0.366 & 0.351 & 0.383 & 0.800 & 0.441 \\
ACT26 & 0.343 & 0.432 & 0.378 & 0.430 & 0.476 & 0.905 \\
ACT27 & 0.370 & 0.537 & 0.384 & 0.467 & 0.471 & 0.924 \\
ACT28 & 0.315 & 0.447 & 0.403 & 0.462 & 0.523 & 0.941 \\
ACT29 & 0.314 & 0.486 & 0.372 & 0.422 & 0.436 & 0.928 \\
\hline & & & & & &
\end{tabular}

\section{Referencias}

Ambrosini, V. \& Bowman, C. (2001). Tacit knowledge: Some suggestions for operationalization. Journal of Management Studies, 38(6), 811-829. https://doi.org/10.1111/1467-6486.00260

Amiryany, N., Ard-Pieter, H. \& Cloodt, M. (2012). Acquisition reconfiguration capability. European Journal of Innovation Management, 15 (2), 177-191. http://dx.doi.org/10.1108/14601061211220

Archibugi, D. \& Pietrobelli, C. (2003). The globalisation of technology and its implications for developing countries: Windows of opportunity or further burden. Technological Forecasting and Social Change, 70(9), 861-883. http:// dx.doi.org/10.1016/S0040-1625(02)00409-2

Arnett, D. B. \& Wittmann, C.M. (2014). Improving marketing success: The role of tacit knowledge exchange between sales and marketing. Journal of Business Research, 67,324-331. http://dx.doi.org/10.1016/j.jbusres.2013.01.018

Arrow, K. J. (1974). The limits of organization. New York, NY: Norton.

Barclay D., Higgins, C. \& Thompson, R. (1995). The partial least squares approach to causal modeling: personal computer adoption and use as an illustration. Technology Studies, 2(2), 285-309.

Bennett, R. (1999). Foreign marketing control decisions of firms engaged in west east technology transfer: A test of the transactions cost hypothesis. International Journal of Technology Management, 17(4). 402- 420. http://dx.doi. org/10.1504/IJTM.1999.002721 
Berchicci, L. (2013). Towards an open R\&D system: Internal R\&D investment, external knowledge acquisition and innovative performance. Research Policy, 42(1), 117-127. https://doi.org/10.1016/j.respol.2012.04.017

Boiral, O. (2002). Tacit knowledge and environmental management. Long Range Planning, 35(3), 291-317. https://doi. org/10.1016/s0024-6301(02)00047-x

Chin, W. W. (1998). The partial least squares approach to structural equation modeling. En G. Marcoulides (Ed.). Modern Methods for Business Research (pp.295-336). Mahwah, NJ: Lawrence Erlbaum.

Chin, W. W. \& Newsted, P. R. (1999). Structural equation modeling analysis with small samples using partial least squares. Statistical strategies for small sample research, Thousand Oaks, CA: Sage Publications; 307-341.

Cohen, W. M., Levinthal, D. A. (1989). Innovation and learning: the two faces of R \& D. The Economic Journal, 99(397), 569-596. https://doi.org/10.2307/2233763

Collins, C. J. \& Smith, K. G. (2006). Knowledge exchange and combination: The role of human resource practices in the performance of high-technology firms. Academy of Management Journal, 49(3), 544-560. https://doi. org/10.5465/amj.2006.21794671

Cooper, C. (2006). Knowledge management and tourism. Annals of Tourism Research, 33(1), 47-64. https://doi.org/10.1016/j.annals.2005.04.005

Cronbach, L.J. (1951). Coefficient alpha and the internal structure of test. Psychometrika, 16, september, 197-334. https://doi.org/10.1007/bf02310555

Davenport, T. H., \& Prusak, L. (2000). Working knowledge: how organizations manage what they know. Boston MA: Harvard Business School Press. https://doi.org/10.5860/choice.35-5167

Davenport, T. H. \& Prusak, L. (2001). Conocimiento en acción. Cómo las organizaciones manejan lo que saben. (1 ${ }^{\mathrm{a}}$. Ed.) Buenos Aires: Prentice Hall.

Delić, D. \& Lenz, H-J. (2008). Benchmarking user perceived impact for web portal success evaluation. JIOS, 32 (1), 1-14. Disponible en: http://jios.foi.hr/index.php/jios/article/view/53/47

Desouza, K.C., Awazu, Y. \& Wan, Y. (2006). Factors governing the consumption of explicit knowledge. Journal of the American Society for Information Science and Technology, 57(1), 36-43. http://dx.doi.org/10.1002/asi.20250

Dhanaraj, C., Lyles, M. A., Steensma, H. K. \& Tihanyi, L. (2004). Managing tacit and explicit knowledge transfer in IJTs: The role of relational embeddedness and the impact on performance. Journal of International Business Studies, 35(5), 428-442. https://doi.org/10.1057/palgrave.jibs.8400098

Dyer, J. H. \& Hatch, N. W. (2006). Relation-specific capabilities and barriers to knowledge transfers: creating advantage through network relationships. Strategic Management Journal, 27(8), 701-719. https://doi.org/10.1002/smj.543

Dirks, K. T. \& Ferrin, D. L. (2001). The role of trust in organizational settings. Organization Science, 12(4), 450-467. https://doi.org/10.1287/orsc.12.4.450.10640

Duarte P. A. O. \& Raposo M. L. B. (2010). A PLS Model to Study Brand Preference: An Application to the Mobile Phone Market. Esposito Vinzi et al. (eds.) Handbook of Partial Least Squares, Springer Handbooks of Computational Statistics. November, 449-485. http://dx.doi.org/10.1007/978-3-540-32827-8_21

Easterby-Smith, M. \& Prieto, I. M. (2008). Dynamic capabilities and knowledge management: an integrative role for learning?. British Journal of Management, 19(3), 235-249.https://doi.org/10.1111/j.1467-8551.2007.00543.x

Flores-Ortiz, M. V., Vega-López, A. \& Chávez-Moreno, E. A. (2015). Factores de contingencia que inciden en la profesionalización de las empresas familiares del sector comercio en Tijuana, BC, México. Revista Internacional Administración y Finanzas, 8(3), 15-29.

Fornell, C. \& Larcker, D. (1981). Evaluating structural equation models with unobservable variables and measurement error. Journal of Marketing Research, 18(1), 39-50. https://doi.org/10.2307/3151312

Garson, G. D. (2010). Multiple Regression. Statnotes: Topics in Multivariate Analysis de North Carolina State University. Disponible en http://faculty.chass.ncsu.edu/garson/PA765/regress.htm\#multicollinearity

Gjelsvik, M. (2002). Hotels as Learning Arenas. Scandinavian Journal of Hospitality and Tourism, 2 (1), 31-48. http:// dx.doi.org/10.1080/150222502760347527.

Grant, R. M. (1996). Toward a knowledge-based theory of the firm. Strategy Management Journal, 17, 109-122. https://doi.org/10.1002/smj.4250171110

Gummesson, E. (2017a). From relationship marketing to total relationship marketing and beyond. Journal of Services Marketing, 31(1), 16-19. https://doi.org/10.1108/jsm-11-2016-0398 
Gummesson, E. (2017b). Case theory in business and management: reinventing case study research. London: Sage. https://doi.org/10.4135/9781473920811

Hair, J. F., Jr., Anderson, R. E., Tatham, R. L. \& Black, W. C. (1998). Multivariate data analysis (5th ed.). London: Prentice Hall International.

Hair, J.F.; Bush, R.P. \& Ortinau, D.J. (2010). Investigación de Mercados: en un ambiente de información digital. $4^{\mathrm{a}}$ ed. Mexico: MacGraw-Hill.

Harvey, J. (2012). Managing organizational memory with intergenerational knowledge transfer, Journal of Knowledge Management, 16(3), 400-417. http://dx.doi.org/10.1108/13673271211238733.

Henseler, J., Ringle, C. M. \& Sinkovics, R. R. (2009). The use of partial least squares path modeling in international marketing. Advances in International Marketing. 20, 277-319. http://dx.doi.org/10.1108/S14747979(2009)0000020014.

Hjalager, A. M. (2002). Repairing innovation defectiveness in tourism. Tourism Management, 23(5), 465-474. https:// doi.org/10.1016/s0261-5177(02)00013-4

Huang, C. M., Chang, H. C. \& Henderson, S. (2008). Knowledge transfer barriers between research and development and marketing groups within Taiwanese small-and medium-sized enterprise high-technology new product development teams. Human Factors and Ergonomics in Manufacturing \& Service Industries, 18(6), 621-657. https:// doi.org/10.1002/hfm.20130

Hulland, J. (1999). Use of partial least squares (PLS) in strategic management research: a review of four recent studies. Strategic Management Journal, 20(2), 195-204. http://dx.doi.org/10.1002/(SICI)1097-0266(199902).

Hunt, S. D., Arnett, D. B. \& Madhavaram, S. (2006). The exploratory foundations of relationship marketing theory. The Journal of Business and Industrial Marketing, 2(1/2), 72-87. http://dx.doi.org/10.1108/10610420610651296].

Inkpen, A. (2008). Managing KT in international alliances. Thunderbird International Business Review, 50 (2), 77-90. https://doi.org/10.1002/tie.20180

Instituto Nacional de Estadística e Informática [INEGI] (2013). Sistema de Cuentas Nacionales de México. Disponible en: www.inegi.org

Jafari, M. \& Mozhden, A. (2013). Classification of human resources based on measurement of tacit knowledge. Journal of Management Development, 32 (4), 376-403. http://dx.doi.org/10.1108/02621711311326374

Johnson, M. P. (2017). Knowledge acquisition and development in sustainability-oriented small and medium-sized enterprises: Exploring the practices, capabilities and cooperation. Journal of Cleaner Production, 142, 3769-3781. https://doi.org/10.1016/j.jclepro.2016.10.087

Kale, P., Singh, H. \& Perlmutter, H. (2000). Learning and protection of proprietary assets in strategic alliances: building relational capital. Strategic Management Journal, 21(3), 217-237. http://dx.doi.org/10.1.1.607.7854yrep=replytype $=$ pdf

Kang, S. C., Morris, S. S. \& Snell, S. A. (2007). Relational archetypes, organizational learning, and value creation: Extending the human resource architecture. Academy of Management Review, 32(1), 236-256. https://doi. org/10.5465/amr.2007.23464060

Kenny, D.A. (2015). Mediation. Disponible en http://davidakenny.net/cm/mediate.htm. https://doi. org/10.1002/9781118445112.stat06605

Kucharska, W. \& Kowalczyk, R. (2016). Trust, collaborative culture and tacit knowledge sharing in project management-a relationship model. Proceedings of the 13th international conference on intellectual capital, knowledge management \& organizational learning: ICICKM 2016, 159-166. DOI: 10.13140/RG.2.2.25908.04486

Lane, P.J., Koka, B.R. \& Pathak, S. (2006). The reification of absorptive capacity: a critical review and rejuvenation of the construct. Academy of Management Review, 31 (4), 833-863. https://doi.org/10.5465/amr.2006.22527456

Liao, Y. \& Barnes, J. (2015). Knowledge acquisition and product innovation flexibility in SMEs. Business Process Management Journal, 21(6), 1257-1278. https://doi.org/10.1108/bpmj-05-2014-0039

Liao, S. H., Chang, W. J., Hu, D. C. \& Yueh, Y. L. (2012). Relationships among organizational culture, knowledge acquisition, organizational learning, and organizational innovation in Taiwan's banking and insurance industries. The International Journal of Human Resource Management, 23(1), 52-70. https://doi.org/10.1080/09585192.201 1.599947 
Lin, C. P. (2007). To share or not to share: Modeling tacit knowledge sharing its mediators and antecedents. Journal of Business Ethics, 70(4), 411-428. http://dx.doi.org/10.1007/s10551-006-9119-0.

Ma, J., Choi, B. \& O'Connor, G. (2016). Marketing's role in capturing value from innovation: Knowledge resources, strategic emphasis, and firm value. In Management of Engineering and Technology (PICMET), 2016 Portland International Conference on IEEE, 1271-1277. https://doi.org/10.1109/picmet.2016.7806634

Massey, G. R. \& Dawes, P.L. (2007). Personal characteristics, trust, conflict, and effectiveness on marketing/sales working relationships. European Journal of Marketing, 41(9/10), 1117-1145. https://doi.org/10.1108/03090560710773372

Maurer, I. (2010). How to build trust in inter-organizational projects: The impact of project staffing and project rewards on the formation of trust, knowledge acquisition and product innovation. International Journal of Project Management, 28(7), 629-637. https://doi.org/10.1016/j.ijproman.2009.11.006

Mirc, N. (2013). Human impacts on the performance of mergers and acquisitions. Advances in Mergers and Acquisitions, 12, December, 1-31. http://dx.doi.org/10.1108/S1479-361X(2013)0000012004

Mohamad, O., Ramayah, T. \& Hathaivaseawong, N. (2010). Transfer of marketing knowledge in Thai international joint venture firms. Asian Academy of Management Journal, 15(2), 197-216.

Morgan, R. M. \& Hunt, S. D. (1994). The commitment -trust theory of relationship marketing. The Journal of Marketing, 58(3), 20-38. https://doi.org/10.2307/1252308

Nahapiet, J. \& Ghoshal, S. (1998). Social capital, intellectual capital, and the organizational advantage. Academy of Management Review. 23(2). 242-266. https://doi.org/10.5465/amr.1998.533225

Nonaka, I. \& Takeuchi, H. (1995). The knowledge-creating company: How Japanese Companies Create the Dynamics of Innovation. New York-Oxford: Oxford University Press. https://doi.org/10.1016/0024-6301(96)81509-3

Rodríguez Antón, J., Oliva, F. \& Laguna, M. (2003). Cambio organizativo y gestión del conocimiento en el sector turismo. XII Congreso AECA. Disponible en: https://www.google.com.mx/?gws_rd=ssl\#q=Cambio+organizativo+y+-gesti\%C3\%B3n+del+conocimiento+en+el+sector+turismo\%2C+Comunicaci\%C3\%B3n+presentada+al+XII+-Congreso+AECA. (fecha de consulta: 12 de marzo 2016)

Ruggles, R. (1998). The state of the notion: Knowledge management in practice. California Management Review, 40(3), 80-89. http://dx.doi.org/10.2307/41165944.

Secretaría de Turismo [SECTUR]. (2014). Sector Turismo, el mayor generador de empleos. Disponible en www. sectur.gob.mx

Seidel, G. \& Back A. (2009). Success factor validation for global Erp.17th. European Conference on Information Systems. Research Paper Manuscript. ID: ECIS2009-0098.R1. Submission Type: Research Paper. Disponible en: https://www.alexandria.unisg.ch/213715/1/ecis2009-0098.pdf (fecha de consulta 26 de marzo del 2016)

Simonin, B. L. (1999). Ambiguity and the process of knowledge transfer in strategic alliances. Strategic Management Journal, 20(7), 595-623. http://dx.doi.org/10.1002/(SICI)1097-0266(199907)

Sistema Integral de Información de Mercados Turísticos (2014). Inteligencia de Mercados. Sector turístico en México. Disponible en: http://www.siimt.com (fecha de consulta 26 de marzo del 2016)

Swan, J. I., Trawick Jr., F., Rink, D. \& Roberts, J. (1988). Measuring dimensions of purchaser trust of industrial salespeople. Journal of Personal Selling \& Sales Management, 8(1), 1-9. URL: http://www.jstor.org/stable/20832436

Szulanski, G. (2000). The process of knowledge transfer: A diachronic analysis of stickiness. Organizational Behavior and Human Decision Processes, 82(1), 9-27. https://doi.org/10.1006/obhd.2000.2884

Tenenhaus, M., Vinzi, V.E., Chatelin, Y.-M. \& Lauro C. (2005). PLS pathmodeling. Computational Statistics and Data Analysis, 48(1), 159-205. http://dx.doi.org/10.1016/j.csda.2004.03.005.

Thiétart, R. A. (2001). Doing management research: a comprehensive guide. Sage Publications Ltd. France.

Tsai, W. \& Ghoshal, S. (1998). Social capital and value creation: The role of intrafirm networks. Academy of management Journal, 41(4), 464-476. https://doi.org/10.2307/257085

Wiewiora, A., Trigunarsyah, B., Murphy, G. \& Coffey, V. (2013). Organizational culture and willingness to share knowledge: A competing values perspective in Australian context. International Journal of Project Management, 31(8), 1163-1174. https://doi.org/10.1016/j.ijproman.2012.12.014

Yang, J. T. \& Wan, C. S. (2004). Advancing organizational effectiveness and knowledge management implementation. Tourism Management, 25(5), 593-601. http://dx.doi.org/10.1016/j.tourman.2003.08.002. 
http://dx.doi.org/10.22201/fca.24488410e.2018.1364

Yli-Renko, H., Autio, E. \& Sapienza, H. J. (2001). Social capital, knowledge acquisition, and knowledge exploitation in young technology-based firms. Strategic Management Journal, 22(6-7), 587-613. https://doi.org/10.1002/smj.183

Young, C. L. \& Wilkinson, I. F. (1989). The role of trust and co-operation in marketing channels: A preliminary study. European Journal of Marketing, 23(2), 109-121. http://dx.doi.org/10.1108/EUM0000000000550.

Zahra, S. A. \& George, G. (2002). Absorptive capacity: A review, reconceptualization, and extension. Academy of Management Review, 27(2), 185-203. https://doi.org/10.2307/4134351

Zapata, L, E. (2004). Los determinantes de la generación y la transferencia del conocimiento en pequeñas y medianas empresas del sector de las tecnologías de la información de Barcelona. Tesis Doctoral. Universidad Autónoma de Barcelona, Bellaterra, España. Disponible en http://hdl.handle.net/10803/3955 